\title{
DIVERGENT VIEWS
}

\section{Enzyme nomenclature: Functional or structural?}

\author{
PETER GEGENHEIMER \\ Section of Biochemistry and Biophysics, Department of Molecular Biosciences, and Molecular Genetics Program, \\ The University of Kansas, Lawrence, Kansas 66045-7534, USA
}

Keywords: catalytic mechanism; enzyme nomenclature; ribonuclease P; ribozyme

\section{FUNCTION-BASED VERSUS STRUCTURE-BASED NOMENCLATURE}

Altman and colleagues (this issue) call attention to the inability of current standardized enzyme nomenclature to distinguish between enzymatic activities that reside in nonhomologous macromolecules. This issue is highlighted by the fact that the pre-tRNA 5'-maturation activities of bacteria and plant chloroplasts present the first instance (of which I am aware) of two naturally occurring enzymes that cannot be evolutionarily related, but which catalyze an identical reaction. (In the classic example of convergent evolution between the trypsin family and subtilisin, the enzymes do not have an identical substrate specificity.) Altman and colleagues propose that a single trivial name be used only for members of a family of homologous macromolecules; in other words, that different trivial names be given to enzymes that catalyze the same precursor-product conversion but do so with different catalytic mechanisms, or which are not members of a single family of homologous macromolecules.

I am not convinced that there is a problem needing solution. The current proposal seems to run counter to the rationale behind current $E C$ nomenclature, and could create more confusion than it would alleviate.

One can distinguish between a function-based nomenclature based on the biochemical reaction catalyzed-the substrate-product conversion-and a structure-based nomenclature based on the physical nature of the catalyst. For a classical enzymologist, the reaction type being catalyzed is paramount: It is the reaction that one uses to purify the enzyme. One identifies the enzyme based on its activity, whereas its physical structure may initially be of secondary importance. The value of function-based nomenclature is precisely

Reprint requests to: Peter Gegenheimer, Department of Molecular Biosciences, The University of Kansas, 1200 Sunnyside Ave., Lawrence, Kansas 66045-7534, USA; e-mail: pgegen@ku.edu. that it allows the biochemical reaction (the substrateproduct conversion) to be described, specified, and studied concomitant with continuing purification and analysis of the corresponding enzyme. Further, as more is learned about the enzyme's structure and catalytic mechanism, it is not necessary to rename it. Indeed, the utility of function-based nomenclature is exemplified by the history of bacterial RNase P purification and characterization.

\section{CANONICAL AND NON-CANONICAL RNase P}

The prototype RNase $\mathrm{P}$ was purified by Robertson et al. (1972), who determined that the enzyme made a single endonucleolytic cleavage in a $5^{\prime}$-extended precursor to Escherichia coli tRNA ${ }^{\mathrm{Tyr}}$, releasing a $3^{\prime}$-hydroxylterminated leader RNA and generating a 5'-mature tRNA ${ }^{\text {Tyr }}$ with the correct 5'-phosphoryl terminus. The activity was named RNase $P$ because it acted upon precursor tRNA: "These results [the product characterization] indicate that the enzymatic activity which we are studying here has a simple mode of action on the precursor: we designate this activity ribonuclease $P$. We can further conclude that RNase $P$ purified to this stage is already free of the activity or activities which degrade the 5 '-terminal fragment and remove the extra nucleotides from the $3^{\prime}$ end of the precursor" (Robertson et al., 1972; p. 5247). At the time of discovery, the polyanionic nature of RNase $P$ was noted, but it was not until 6 years later that the RNA subunit was discovered (Stark et al., 1978), 2 more years before it found to be required in vivo (Kole et al., 1980), and another 3 years before the RNA was shown to be the catalytic component (Guerrier-Takada et al., 1983). During the 11 years from identification of the reaction to identification of the catalyst, the designation "RNase P" proved useful because it was a function-based and not a structure-based name.

The definition of EC 3.1.26 is "Hydrolases; Acting on ester bonds; Endoribonucleases producing 5'- 
phosphomonoesters." The current definition of RNase P, EC 3.1.26.5, is: "Recommended name: ribonuclease P; Reaction: Endonucleolytic cleavage of RNA, removing 5'-extranucleotides from tRNA precursor; Comments: An RNA-containing enzyme, essential for tRNA processing; generates 5 '-termini of mature tRNA molecules" (NC-IUBMB, 1992; p. 342; Schomburg \& Salzmann, 1991). Clearly, these reaction specifications identify any pre-tRNA 5'-maturation endoribonuclease that acts as a true hydrolase rather than a phosphoryltransferase. The possession of an RNA subunit is not a formal part of the enzyme definition.

\section{CURRENT NOMENCLATURE GUIDELINES}

Current IUPAC guidelines make it clear that enzymes are named after the reaction catalyzed, and acknowledge the possibility that two structurally dissimilar enzymes could catalyze a single reaction: "The second general principle is that enzymes are principally classified and named according to the reaction they catalyze. The chemical reaction catalyzed is the specific property that distinguishes one enzyme from another, and it is logical to use it as the basis for the classification and naming of enzymes" (NC-IUBMB, 1992; p. 5); ". . . the formation of intermediate complexes of the reactants with the enzyme is not taken into account, but only the observed chemical change produced by the complete enzyme reaction" (p. 6). "A second consequence of this concept is that a certain name designates not a single enzyme protein but a group of proteins with the same catalytic property" (p. 6).

Altman and colleagues correctly point out that EC recommendations allow for variances from reactionbased nomenclature. "However, there are exceptions to this general rule. Some are justified because the mechanism of the reaction or the substrate specificity is so different as to warrant different entries in the enzyme list" (NC-IUBMB, 1992, p. 6). This variance might be justified once the reaction mechanism of a nonribozyme RNase $\mathrm{P}$ is known. A second condition is: "The chemical nature of the enzyme has, however, been used exceptionally in certain cases where classification based on specificity is difficult, for example, with the peptidases (subclass 3.4)" (p. 5). This seems meant for use in determining the reaction class when other indicators fail. For the RNase Ps, however, one cannot make the case that the substrate specificity or precursor-product relationships are not well established or are difficult to determine.

\section{CLASSIFICATION OF NONCANONICAL RNase P ACTIVITIES}

Altman et al. are concerned lest a specific enzyme name be attached to an insufficiently well-defined physical entity. However, even in early reports, the spinach chloroplast enzyme was sufficiently purified for a definitive assignment to be made of the substrate-product relationship (Wang et al., 1988), and hence for assignment of a semisystematic name ("pre-tRNA 5'maturation endonuclease"). Furthermore, the reaction (substrate-product conversion) was shown to be identical to the prototype RNase $P$ reaction, and on that basis, the trivial name RNase $P$ was adopted (Wang et al., 1988; p. 1572): "Plant chloroplasts contain a tRNA $5^{\prime}$ maturation activity which is the functional analogue of eubacterial RNase P. We designate the chloroplast activity as RNase $\mathrm{P}$ based solely on the reaction catalyzed. Both activities cleave tRNA precursors precisely at the $5^{\prime}$ side of the mature tRNA domain, releasing a single leader RNA fragment and exposing the mature tRNA 5' terminus." The physical differences between this protein enzyme and the canonical ribozyme-type RNase $\mathrm{P}$ were established by Wang et al. (1988) and have been amplified by further purification and analysis (e.g., Thomas \& Gegenheimer, 2000). More purified preparations clearly lack any contaminating RNA fragments (Li, 1992) and contain identifiable polypeptides (Thomas \& Gegenheimer, 2000).

\section{A LITMUS TEST FOR RNase P?}

Altman and colleagues pose several questions about the relationship between noncanonical (non-RNAbased) pre-tRNA 5'-maturation activities and the canonical RNA-based activity:

First and second, are the substrate specificities the same? Are the chemical reaction mechanisms identical? These are not prerequisite for being an RNase P: substrate specificities differ among bacterial, archaeal, and nuclear RNases Ps, in particular with respect to the requirement for a CCA terminus and in the utilization of non-pre-tRNA substrates. By definition, a pre-tRNA $5^{\prime}$-maturation endonuclease that can act on homologous substrates (from the same organism and organelle) has the required, physiologically appropriate, specificity. Reaction mechanism can be used to differentiate among similar enzymes when the mechanism is much different-and when it is definitively established.

Third and fourth, have completely purified activities been studied? And has their physiological role been established genetically? The first criterion is relevant to nomenclature only insofar as it is necessary that an enzyme name be given to a single biochemical entity rather than to a multienzyme complex (NC-IUBMB, 1992; p. 5). Most enzyme activities have been named prior to their purification to homogeneity. Granted, the burden is upon subsequent researchers to prove that their activities conform to the prototype. But this simply means establishing that the newer activities catalyze the identical substrate-product transformation. The second criterion, while of obvious importance, has never 
played a role in biochemical nomenclature. One can specify and analyze an enzyme without knowing its physiological role, much as one can specify and analyze a gene without knowing its encoded product.

The fact that the bacterial, archaeal, and nuclear RNase P RNA subunits are homologous, and that many of the archaeal and nuclear protein subunits may be homologous, has little bearing on nomenclature. For example, Altman et al. discuss, as apparently authentic RNase Ps, enzymes from bacteria, archaea, and nuclei whose in vivo role has been established only by structural similarity to the authenticated $E$. coli or yeast nuclear enzymes. Likewise, the possible scenarios whereby an all-protein RNase $\mathrm{P}$ might have arisen are relevant to nomenclature only if it is agreed that enzyme classification should be structure- rather than function-based. On the other hand, enzyme nomenclature based upon the reaction catalyzed rather than upon the evolutionary history of the enzyme allows precise naming to be made in the background of such uncertainty. As the IUPAC points out (Moss, 2000; para. 3), "It is perhaps worth noting, as it has been a matter of long-standing confusion, that enzyme nomenclature is primarily a matter of naming reactions catalysed, not the structures of the proteins that catalyse them. This has allowed assignment of newly discovered catalytic activities before anything is known about the structures of the enzymes."

\section{SOLUTIONS}

The essential question is really whether RNase $P$ is a trivial name for all pre-tRNA 5'-maturation endonucleases, or should designate only those which are homologous to the bacterial enzyme. A practical solution is contained in the current guidelines: "In the light of the fact that enzyme names and code numbers refer to reactions catalyzed rather than to discrete proteins, it is of special importance to give also the source of the enzyme for full identification ..." (NC-IUBMB, 1992; p. 8). Because this is the current practice, it seems simplest to continue doing so.

I favor the conservative approach of following IUPAC guidelines in (1) naming an enzyme after the reaction catalyzed; hence "pre-tRNA 5'-maturation endonuclease"; (2) continuing the use of "RNase P" as a trivial name for this class of reactions; and (3) discriminating among mechanistically or structurally different RNases $P$ by qualifying them with the name of the class of organism from which they are isolated. Hence we can easily and unambiguously differentiate among "bacterial RNase P," "archaeal RNase P," "nuclear RNase P," "fungal mitochondrial RNase P," and "plant chloroplast RNase P." If one states that plant and yeast mitochondria possess a bacterial-type RNase $P$, or proposes that nuclear RNase $P$ evolved from the archaeal enzyme, one immediately knows which enzyme structures are meant. When the biochemical nature of the enzyme is less well-understood, such a statement defines the biochemical transformation catalyzed, and presents the best current understanding of the structure and function of the underlying catalyst.

As more is learned about these activities and their structural and evolutionary relationships, it may prove useful to group them into larger families based primarily on mechanism, as for the protease families. The prototype enzyme activity might be "ribozyme RNase P"; any all-protein RNase P could be called "nonribozyme" RNase P; and if warranted, the archaeal and nuclear enzymes could be designated "hybrid RNase P."

\section{ACKNOWLEDGMENTS}

I thank Profs. Richard Schowen and Richard Himes for advice and discussion, and Dr. Schowen for an alternative nomenclature suggestion.

\section{REFERENCES}

Guerrier-Takada C, Gardiner KJ, Marsh TL, Pace N, Altman S. 1983. The RNA moiety of ribonuclease $P$ is the catalytic subunit of the enzyme. Cell 35:849-857.

Kole R, Baer MF, Stark BC, Altman S. 1980. E. coli RNase P has a required RNA component in vivo. Cell 19:881-887.

Li X. 1992. Precursor tRNA recognition by a novel chloroplast RNase P. Ph.D. dissertation, University of Kansas, Lawrence, Kansas.

Moss GP, ed. 2000. Enzyme nomenclature, in "IUPAC-IUBMB Biochemical Nomenclature Committees," http://www.chem.qmw.ac. uk/iupac/jcbn/\#6.

NC-IUBMB (Nomenclature Committee of the International Union of Biochemistry and Molecular Biology). 1992. Enzyme Nomenclature 1992. San Diego: Academic Press. (EC 3.1.26.5 is defined online at <http://www.chem.qmw.ac.uk/iubmb/enzyme/EC3/ 0121 p.html\#2605 $>$ and the rules are given at $<$ http://www.chem. qmw.ac.uk/iubmb/enzyme/rules.html >.)

Robertson HD, Altman S, Smith JD. 1972. Purification and properties of a specific Escherichia coli ribonuclease which cleaves a tyrosine transfer ribonucleic acid precursor. J Biol Chem 247:52435251.

Schomburg D, Salzmann M., eds. 1991. Enzyme handbook. Vol. 3. Berlin: Springer-Verlag. (Available on-line as the BRENDA database, http://srs.ebi.ac.uk/srs5bin/cgi-bin/wgetz?-e+[BRENDAECNumber:'3.1.26.5']).

Stark BC, Kole R, Bowman EJ, Altman S. 1978. Ribonuclease P: An enzyme with an essential RNA component. Proc Natl Acad Sci USA 75:3717-3721.

Thomas BC, Gegenheimer P. 2000. Chloroplast RNase P does not utilize the ribozyme-type cleavage mechanism. RNA 6:545-553.

Wang M-J, Davis NW, Gegenheimer P. 1988. Novel mechanisms for maturation of chloroplast transfer RNA precursors. EMBO J 7:1567-1574. 

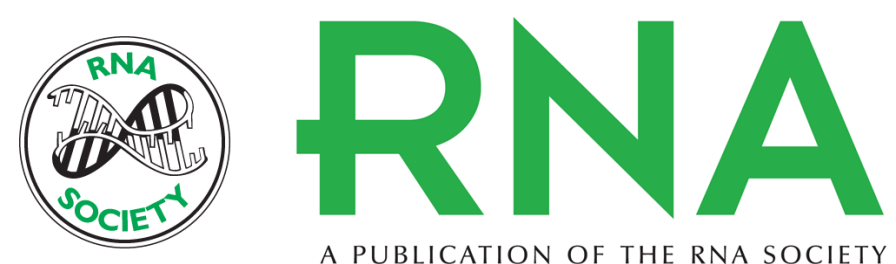

A PUBLICATION OF THE RNA SOCIETY

\title{
Enzyme nomenclature: functional or structural?
}

\author{
P Gegenheimer
}

RNA 2000 6: 1695-1697

Email Alerting Receive free email alerts when new articles cite this article - sign up in the box at the top Service right corner of the article or click here.

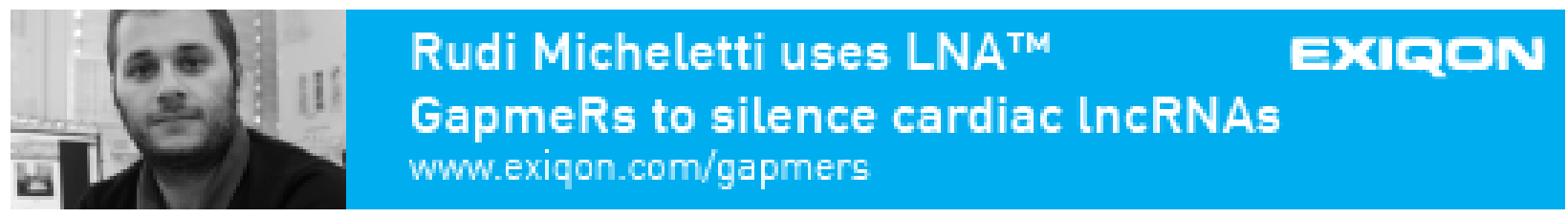

To subscribe to RNA go to: http://rnajournal.cshlp.org/subscriptions 